\title{
Correspendence
}

\section{Comment on "Effect of Artemisia annua and Artemisia afra tea infusions on schistosomiasis in a large clinical trial"}

To the editor,

We read the article entitled "Effect of Artemisia annua and Artemisia afra tea infusions on schistosomiasis in a large clinical trial" by Munyangi et al. with great interest. This trial was presumably designed as a phase III clinical trial with a randomized, controlled, double-blind study that aimed to demonstrate the superiority of $A$. annua and A. afra plant-based infusions over praziquantel for the treatment of schistosomiasis.

We would like to offer a critical analysis of this trial, as we believe there are several crucial issues regarding its scientific background, design, and statistical methods. These concerns question the scientific validity of the results while raising critical issues regarding ethical aspects.

At first, backgrounds should be précised. We agree with the authors that the use of artemisinin-based combination therapies is a promising alternative for treating schistosomiasis as already suggested in a metaanalysis of 24 randomized trials where the cure rate of oral artesunate (alone or combined with various anti-parasitic agents) was compared with that of praziquantel for schistosomiasis (Villar et al., 2012). Nevertheless, in a sub-analysis of this meta-analysis, artesunate alone ( $4 \mathrm{mg} / \mathrm{kg} /$ day for 3 days) was clearly less effective than praziquantel ( $40 \mathrm{mg} / \mathrm{kg}$ once) (odds ratio $=0.27,95 \%$ confidence interval (CI): $0.13-0.53$ ). The crude cure rates were $33 \%$ and $61.5 \%$ in the artesunate and praziquantel groups, respectively. On another hand, artemisinin derivatives are reportedly efficient against the juvenile form of the parasite (schistosomulae), but not against adults or eggs as reported in experimental models (Sabah et al., 1986). This may explain the prophylactic effect of artemisinin derivatives on the disease, but the effect on adults or eggs is less convincing. Indeed, the efficacy of artemether on worm reduction was above $70 \%$ for schistosomulae aged 14-28 days, but decreased for older parasites.

Taken together these experimental and clinical data do not seem sufficient to support such an approach. Moreover $A$. afra does not contain significant artemisinin, and $A$. annua contains a very low and variable level of this molecule as detailed by the authors in their article. Thus, the use of an Artemisia-based infusion in a phase III clinical trial does not seem to be supported by sufficient data to be considered as a valid alternative to praziquantel.

Secondly, regarding the study protocol, the motivations for the exclusion criteria are unclear. Patients over 60 years-old and pregnant women were excluded. The authors did include school-aged children over the age of six years but did not explain how Artemisia infusion was given. Did the children receive the same treatment as the adults? Was the quantity of the infusion reduced? Praziquantel was prescribed at an unusual dosage of $60 \mathrm{mg} / \mathrm{kg} /$ day for 3 days. Most guidelines, including those from the WHO, recommend $40 \mathrm{mg} / \mathrm{kg}$ in a single dose, even for preschool-aged children (Colley et al., 2014; Coulibaly et al., 2017).
Also, the study did not specify how praziquantel was administered to children. Were the pills crushed or dissolved? There are also no specifications on the concomitant intake of food (especially fat), which significantly increases praziquantel bioavailability and ensures the proper administration of the drug (Olliaro et al., 2014). The evaluation of treatment's efficiency relied on a quantitative analysis of the number of schistosomiasis eggs in stool using the Kato-Katz technique within 0-28 days/weeks after treatment. It is indicated that a double count was performed, but it is not clear if two stool samples were analyzed or if two slides of a single stool sample were analyzed. It is noteworthy that the diagnosis of schistosomiasis is difficult due to the variable kinetics of egg elimination in stool (Berhe et al., 2004; Lindholz et al., 2018). The only way to ensure the reliability of this technique is by repeatedly assessing stool samples. The use of a quantitative method for a unique stool sample, even with two independent lectures, seems extremely precarious to conclude the superiority of the treatment, as was done by the authors. Moreover, the methods used to assess the viability of the eggs are not clearly defined in the Materials and Methods section. Finally, complete clearance of eggs from stool is indeed the test that is recommended to determine patient recovery, but this analyses need to be performed at least 3 months after treatment completion to ensure that the patient is cured (Farrar et al., 2014; Gentilini, 2012).

Third, there are a dozen of critical issues with the statistical methods.

1. The study protocol: The sample size calculation is not consistent with the primary outcome. Moreover, the argument used to circumvent the sample size computation, which explained that some preliminary data were required to compute the sample size, is not correct. In the absence of previous data, it is possible to set a clinically significant target value for the therapeutic effect and to calculate a sample size for this effect size, with the power set $a$ priori. Notwithstanding this limit, the presented sample size calculation does not account for the existence of several treatment groups. A precise CI is indicated, but it is not known for what expected value of the proportion, which is fundamental. The use of three treatment groups is less common, but this can be accounted for when calculating the sample size. Nevertheless, this fundamental aspect of design was not taken into account.

2. The primary outcome is not specified.

3. The double-blind protocol was not conducted appropriately. A double placebo is presented, but the Artemisia tea placebo is probably not a true placebo (it was a brief infusion of the plants used in the Artemisia treatment arm) and it is not stated whether artemisinin or other compounds were present in this pseudo-placebo. Table 1 does not answer this question.

4. The statistical analysis is not in line with current standards. The primary outcome seems to be the reduction of the number of eggs 
found in stool. The authors wrote that a chi-square test and analysis of variance were used to compare the three groups at each time point. It would have helped to use a regression model adapted to compare count, such as a negative binomial or quasi-Poisson model. Indeed, it is not known whether the primary outcome, which is discrete by nature, was treated as a quantitative or a qualitative criterion, which would require different statistical methods. There is also no consideration of the repeated nature of the data. Suitable statistical models should have been used (a mixed model with a random subject effect or a model with a variance-covariance matrix). Alternatively, a Cox model until complete clearance of eggs from stool or a cure rate at a predefined time could have been used.

5. The population and primary outcome: Models could have been used to account for confounding factors related to the large imbalance in gender and age distribution between groups, as displayed in Table 2. Besides, this shows that randomization failed completely, which is sufficient to invalidate the trial as a whole. From a practical point of view, it can be said that there was no randomization. Even the number of patients in each arm is inconstant. Fig. 1 shows 400 patients in the PZQ group, 200 in the A. annua group, and 200 in the $A$. afra group at baseline, and 390, 195, and 195 patients, respectively, with full follow-up. The group sizes calculated from Tables 2 and 3 show 388 patients in the PZQ group, 214 in the $A$. annua group, and 178 in the $A$. afra group. Therefore, the group sizes are inconsistent. Block randomization (100 blocks of 8 patients) cannot explain how more than 200 patients were found in the $A$. annua group.

6. There is no information on the management of missing data.

7. It is not known whether the data were analyzed under intent to treat, per protocol, or received treatment.

8. It is also not known whether the authors wanted to compare three groups or two groups by grouping the two types of Artemisia.

9. The fact that treatment is a plant decoction makes the results difficult to interpret because there are many molecules in different proportions that may differ from one sample to another (see Table 1, where the concentrations between groups are clearly different). As a result, the dosages were not controlled, and it is not clear what dosage was administered to the patients.

10. During the survey, comparisons between the three groups were repeated. As a result, the risk of type I error is inflated. No information on multiple comparisons was given (e.g., the use of the Bonferroni correction), suggesting that this was not taken into account.

11. Although not stated in the methods we guess reduction of the number of eggs in stool was the main outcome measure. However conclusions cannot be made about the outcome as there were no statistical testings (no formal test, no $P$-value, no effect size measure, no estimate of an effect in each group, no CI). The comparison is based solely on reduction of the absolute numbers of eggs presented at each time point in a graph.

12. No estimation of within- or between-subject variability across time points was given, either numerically or graphically. Fig. 2 does not provide this information. CIs for each group at each time should have been given. Not taking this into account prevents the authors from formally concluding that eggs were eliminated occurred earlier in the first group, although they do make this claim.

Unsurprisingly, awkward methods led to questionable results. One figure shows a "survival analysis", but this analysis was not described in the Materials and Methods section. This figure also refers to a log-rank test that appears to have been not performed and confuses the name of the test (the log-rank test) with the name of the graphical representation of the survival curves (the Kaplan-Meier method). The observed cure rates in the praziquantel group at 21 days (0\%) and 28 days $(100 \%)$ are hardly believable for 400 (or 388) patients, as physiopathological variance is expected and treatment adherence is rarely $100 \%$. The same pattern is observed at days 3 and 7 in the two Artemisia groups. Furthermore, it is highly unlikely that the two Artemisia groups had exactly the same results, as presented in Figs. 2 and 3. Furthermore, Figs. 2 and 3 are inconsistent. The so-called survival curves of the Artemisia groups fall at day 7, but Fig. 2 shows 6.5 eggs (mean? median? sum?) at day 7.

We also found many inconsistencies between the text, tables and figures.

- The "adverse effects" section reports no adverse effects in any of the Artemisia arms, but Table S1 shows 79 adverse effects in the two pooled Artemisia groups. Moreover, the text specifies vomiting in $26.5 \%$, abdominal pain in $18.5 \%$, and headache in $15.5 \%$ of PZQ patients, while Table S1 reports respective values of 3.8\%, 44.9\%, and $33.3 \%$.

- The text specifies that $28 \%, 12 \%$, and $12 \%$ of patients, had melena at baseline in the PZQ, A. annua, and A. afra groups, respectively. The rates computed from Table 3 are $56.4 \%, 42.5 \%$, and $52.2 \%$, respectively.

- At day 7, Fig. 2 show 6.5 eggs, on average, in the two Artemisia groups, while Fig. 3 shows $0 \%$ egg survival. The text claims that the average numbers of eggs at baseline were 739, 748, and 726 for PZQ, A. annua, and A. afra, while Fig. 2 shows 739.0, 736.0, and 738.1 , respectively. The average egg count at day 3 ( 6 per patient in the text, but 66 in Fig. 2) is also inconsistent for A. afra and A. afra. Moreover, identical egg counts were found at day 3 and 7 for $A$. annua and $A$. afra (66 at day 3 and 6 at day 7 for both treatments), which seems unlikely (differences between groups were not statistically tested).

In addition, some data exhibit strange patterns. Table S1 contains many adverse effect frequencies that are multiples of five, with 17 multiples of five for 21 non-zero frequencies. According to a binomial distribution, the probability of 17 or more multiples of five for 21 frequencies occurring by chance is approximatively $3.4 \times 10^{-9}$. Moreover, the difference between adverse events and adverse effects (adverse events attributable to treatment) is never mentioned. The very high rates of adverse effects similar to schistosomiasis symptoms in the PZQ arm suggest that all adverse events may have been presented. The very low rate of schistosomiasis-related adverse events in the Artemisia arms suggests a differential declaration bias, implying that blindness to treatment was broken.

In conclusion, a critical analysis of this clinical trial leads to major concerns regarding its scientific background, methodology, and results. We believe that this study was not conducted to international standards and scientific guidelines. Moreover, the existence of an efficient treatment for schistosomiasis, namely praziquantel, should prevent phase III clinical trials for which the efficacy of the therapeutic alternative has not been proven in vitro, in animal models or through phase I and II clinical trials.

\section{Acknowledgments}

The authors would like to thank Enago (www.enago.com) for the English language review.

\section{References}

Berhe, N., Medhin, G., Erko, B., Smith, T., Gedamu, S., Bereded, D., Moore, R., Habte, E., Redda, A., Gebre-Michael, T., Gundersen, S.G., 2004. Variations in helminth faecal egg counts in Kato-Katz thick smears and their implications in assessing infection status with Schistosoma mansoni. Acta Trop. 92, 205-212. https://doi.org/10.1016/ j.actatropica.2004.06.011.

Colley, D.G., Bustinduy, A.L., Secor, W.E., King, C.H., 2014. Human schistosomiasis Lancet 383, 2253-2264. https://doi.org/10.1016/S0140-6736(13)61949-2.

Coulibaly, J.T., Panic, G., Silué, K.D., Kovač, J., Hattendorf, J., Keiser, J., 2017. Efficacy 
and safety of praziquantel in preschool-aged and school-aged children infected with Schistosoma mansoni: a randomised controlled, parallel-group, dose-ranging, phase 2 trial. Lancet Glob. Health 5, e688-e698. https://doi.org/10.1016/S2214-109X(17) 30187-0.

Farrar, J., Hotez, P., Junghanss, T., Kang, G., Lalloo, D., White, N., 2014. Manson's Tropical Infectious Diseases. 2014.

Gentilini, M., 2012. Médecine Tropicale, 6e éd. Libr. Lavoisier 2012.

Lindholz, C.G., Favero, V., de Verissimo, C.M., Candido, R.R.F., de Souza, R.P., dos Santos, R.R., Morassutti, A.L., Bittencourt, H.R., Jones, M.K., Pierre, T.G.S., GraeffTeixeira, C., 2018. Study of diagnostic accuracy of Helmintex, Kato-Katz, and POCCCA methods for diagnosing intestinal schistosomiasis in Candeal, a low intensity transmission area in northeastern Brazil. PLoS Negl. Trop. Dis. 12, e0006274. https://doi.org/10.1371/journal.pntd.0006274.

Olliaro, P., Delgado-Romero, P., Keiser, J., 2014. The little we know about the pharmacokinetics and pharmacodynamics of praziquantel (racemate and R-enantiomer). J. Antimicrob. Chemother. 69, 863-870. https://doi.org/10.1093/jac/dkt491.

Sabah, A.A., Fletcher, C., Webbe, G., Doenhoff, M.J., 1986. Schistosoma mansoni: chemotherapy of infections of different ages. Exp. Parasitol. 61, 294-303.

Villar del, L.P., Burguillo, F.J., López-Abán, J., Muro, A., 2012. Systematic Review and Meta-Analysis of Artemisinin Based Therapies for the Treatment and Prevention of Schistosomiasis. PLOS ONE 7, e45867. https://doi.org/10.1371/journal.pone.
0045867.

Xavier Argemi $^{\mathrm{a}, *}$, Yves Hansmann ${ }^{\mathrm{a}}$, Jean Gaudart ${ }^{\mathrm{b}}$, André Gillibert ${ }^{\mathrm{c}}$, Eric Caumes ${ }^{\mathrm{d}}$, Stéphane Jauréguiberry ${ }^{\mathrm{e}}$, Nicolas Meyer

${ }^{a}$ Maladies Infectieuses et Tropicales, Université de Strasbourg, CHU de Strasbourg, EA7290, 67000 Strasbourg, France

${ }^{\mathrm{b}}$ Aix Marseille Univ, APHM, INSERM, IRD, SESSTIM, Hop Timone, BioSTIC, Biostatistic \& ICT, 13000 Marseille, France ${ }^{\mathrm{c}}$ Unité de Biostatistique, CHU de Rouen, F 76031 France

${ }^{\mathrm{d}}$ Maladies Infectieuses et Tropicales, CHU Pitié Salpêtrière, APH-HP, Sorbonne Université, 75013 Paris, France

${ }^{\mathrm{e}}$ Maladies Infectieuses et Tropicales, CHU Pitié Salpêtrière, APH-HP, 75013

Paris, France

${ }^{\mathrm{f}}$ GMRC, Service de Santé Publique, Université de Strasbourg, CHU de Strasbourg. iCUBE, UMR7357, 67000 Strasbourg, France E-mail address: xavier.argemi@chru-strasbourg.fr (X. Argemi).

${ }^{*}$ Corresponding author 